CXCR4 with gp120, can cause upregulation of TRAIL receptors and consequent sensitivity to apoptosis by this pathway. In this work, our aim was to measure if CD4+T lymphocytes from HIV+ patients, had and increased expression of TRAIL ligand and death receptors (DR) 5/4 and coreceptor CXCR4 and, evaluate its association

Methods Ten HIV+ ART naïve patients and seven HIVdonors were recruited. The immunophenotyping of $\mathrm{CD} 4+\mathrm{T}$ lymphocytes was performed with CD3+/CD4+ labeling, after that the TRAIL death receptors DR4 and DR5 as well as the TRAIL ligand and the coreceptor CXCR4 were measured for expression and MFI (median fluorescence intensity) by flow cytometry in whole blood samples

Results CD4+T lymphocytes from HIV+ patients showed an augmented expression and MFI of both death receptors and TRAIL ligand compared with the healthy controls; on the other hand, expression of coreceptor CXCR4 was increased in the HIV+ group and the MFI showed a significant difference compared to healthy subjects. Correlations between DR4 and the TRAIL ligand with CXCR4 showed no significance; in contrast, both expression and MFI of DR5 and CXCR5 were significant and showed a strong direct correlation (expression: $\mathrm{p}<0.05 \mathrm{r}=0.69$ and MFI: $\mathrm{p}<0.005 \mathrm{r}=0.81$ ).

Conclusion There is evidence of apoptosis triggered by CXCR4 activation, not related to the Fas pathway, which is one of the main causes related to cell depletion in HIV infection. The upregulation of the TRAIL pathway in HIV infected subjects is correlated with the CXCR4 expression, which could be the cause of the reported apoptosis in these patients. Disclosure No significant relationships.

\section{P170 EFAVIRENZ BASED ANTI-RETROVIRAL REGIMENS IN PAEDIATRICS: A PEEP INTO NEUROPSYCHIATRIC AND BIOCHEMICAL DERANGEMENTS}

'Purba Chakrabarty*, ${ }^{2}$ Manasi Baneriee, ${ }^{2}$ Manab Nandy, ${ }^{3}$ Kalpana Datta, ${ }^{4}$ Rudraprasad Acharya, ${ }^{5}$ Arup Chakraborty, ${ }^{6}$ Pragnadyuti Mandal, ${ }^{6}$ Dipak Sarkar, ${ }^{7}$ Shatavisa Mukherjee, ${ }^{8}$ Mitali Basu, ${ }^{9}$ Ananya Bhowmik. ${ }^{1}$ Medical College and Hospital, Kolkata, 88, College Street, Kolkata - 700073, West Bengal, India, Pharmacology, Dist Bankura, West Bengal, India; ${ }^{2}$ Medical College and Hospital, Kolkata, Pharmacology, Kolkata, India; ${ }^{3}$ Medical College, Kolkata, Paediatrics, Nodal Officer and Programme Director, Paediatric Centre of Excellence in HIV Care, Regional Art Centre, Medical College and Hospital, Kolkata, India; ${ }^{4}$ Medical College and Hospital, Kolkata, Psychiatry, Kolkata, India; ${ }^{5}$ Medical College and Hospital, Kolkata, Community Medicine, Kolkata, India; ${ }^{6}$ Medical College, Kolkata, Pharmacology, Kolkata, India; ${ }^{7}$ School of Tropical Medicine, Kolkata, Patient Safety-pharmacovigilance, ADR Monitoring Centre, Kolkata, India; ${ }^{8}$ Bankura Sammilani Medical College and Hospital, Medicine, Nodal Officer, Fiart Centre, Bankura, India; ${ }^{9}$ Medical College and Hospital, Kolkata, Research Officer, Paediatric Centre Of Excellence in HIV Care, Regional Art Centre, Kolkata, India

10.1136/sextrans-2019-sti.329

Background Combination antiretroviral therapy (ART)has transformed HIV-AIDS into a chronically manageable illness. Efavirenz is an important component for ART regimens. Quality of life should be maintained for the easy continuation of this life-long therapy. Hence the adverse drug reaction (ADR) monitoring has become an important event to achieve good adherence

Objective This study aims to assess the spectrum of clinicobiochemical and neuropsychiatric ADRs for Efavirenz based ART in paediatric HIV patients

Methods Paediatric HIV-1 patients initiated with Efavirenz based ART were followed up for biochemical changes (liver, renal, lipid, haemoglobin parameters) and neuropsychiatric adverse reactions (NPARs) till 6 months. NPAR(s) were monitored using Developmental Psychopathology Checklist and Sleep Disturbances Scale for Children. Adherence and guardian's literacy status were analyzed for their association with the self-reporting of NPAR(s).

Results NPARs were observed to be maximum at $15^{\text {th }}$ day, followed by a gradual decrease over $1^{\text {st }}$ and $2^{\text {nd }}$ month. Symptoms like learning disorder and psychosis were maximum, followed by hyperkinesis, emotional disorder and somatisation. Domains of sleep problems were maximum at $15^{\text {th }}$ day, followed by a graded decrease in the $1^{\text {st }}$ and $2^{\text {nd }}$ month. Self- reporting was found to be $57.14 \%$ and $37.50 \%$ in guardian with secondary and primary education respectively. Decreased adherence was noted among $16.67 \%$ of patients, managed by proper counselling. While biochemical changes like liver function alterations were observed to be maximum at $6^{\text {th }}$ month $(30.39 \%)$ and $4^{\text {th }}$ month $(7.84 \%)$, changes in renal function and lipid parameters were observed to be maximum at $6^{\text {th }}$ month and drop in haemoglobin at $3^{\text {rd }}$ month.

Conclusion Sensitization about $\operatorname{NPAR}(\mathrm{s})$ should be ensured at the time of therapy initiation to warrant adherence and spontaneous reporting in order to prevent resistance and treatment failure. ART induced anaemia, altered lipid profile, liver and kidney function derangements could be life threatening. This study thus upsurges the need for focused pharmacovigilance and therapeutic drug monitoring of ART for safer patient outcomes.

Disclosure No significant relationships.

\section{P171 ZBTBS GENE EXPRESSION IN CD4+ T CELLS AND SYSTEMIC PRO-INFLAMMATORY CYTOKINES IN NAÏVE AND TREATED HIV PATIENTS}

${ }^{1} J u d i t h$ De Arcos Jimenez*, 'Mariana Ruiz Briseño, ${ }^{1}$ Moises Ramos Solano, ${ }^{2}$ Jaime AndradeVillanueva, 'Luz Gonzalez-Hernandez, ${ }^{1}$ Karina Sanchez-Reyes. ${ }^{1}$ Universidad de Guadalajara, Guadalajara, Mexico; ${ }^{2}$ Hospital Civil Fray Antonio Alcalde, Unidad De VIH, Guadalajara, Mexico

\subsection{6/sextrans-2019-sti.330}

Background Chronic inflammation in HIV infection has been associated with accelerated disease progression. Several external host factors contribute to inflammation like, microbial translocation and coinfections; as well as host intrinsic mechanisms; such as immune activation and the regulation of factors that participate in inflammation pathways. Members of the transcriptional factors ZBTB have been associated with repression of proinflammatory cytokines; the objective of this work was to identify if ZBTB repressors could be contributing to cytokines levels decrease in HIV+ ART-treated patients.

Methods CD4+ T cells were isolated from $12 \mathrm{HIV}$ - and 21 $\mathrm{HIV}+$, subclassified as naïve $(\mathrm{n}=8)$ and treated (undetectable for $\geq 1$ year, $n=13$ ). Gene expression of ZBTB2, ZBTB4, ZBTB7B, ZBTB17, ZBTB38, BCL6 and ZNF131 was determinate by qRT-PCR. Cytokine levels were quantified (IL-1 $\beta$, IFN- $\alpha$, IFN- $\gamma$, TNF- $\alpha$, MCP-1, IL-6, IL-8, IL-10, IL-12, IL-17, IL-18, IL-23, IL-33) by flow cytometry in serum. KruskalWallis test was used for statistical analysis.

Results In naïve patients, only $Z B T B 7 B$ showed a significant up-regulation (2.76 fold). BCL6, ZBTB4, ZBTB38 had an increase but, were not significant $(1.01,0.91,0.13$ fold respectively); treated patients showed a significant up-regulation in ZBTB7B, ZBTB4 and ZBTB38 $(2.23,1.13,1.04$ fold respectively) and, $B C L 6$ had 1.02 fold, however, it was no 
significant. ZBTB2, ZBTB17, ZNF131 did not have, all genes were compared to HIV negative subjects. No significant changes were observed in pro-inflammatory cytokines levels, however, in treated patients, IL-1 $\beta$, TNF- $\alpha$, IL-6, IL-10 and IL-12 showed lower levels compared with naïve patients.

Conclusion We showed an increase gene expression of some ZBTBs in HIV+ patients compared with healthy donors, these genes have been related to the inflammatory cytokines suppression; we found that this difference was higher in treated patients compared to naïve patients, this could be related to a decrease in TNF- $\alpha$, IL-6, IL-10 and IL-12 systemic levels.

Disclosure No significant relationships.

\section{P172 ASSOCIATION BETWEEN SHORT-CHAIN FATTY ACIDS PRODUCING BACTERIA AND CD4 T CELLS RECOVERY IN HIV POSITIVE PATIENTS}

${ }^{1}$ Mariana Ruiz Briseño*, 'Sarah Ratkovich-Gonzalez, ${ }^{1}$ Monserrat Alvarez-Zavala, ${ }^{1}$ Alejandra Vega Magaña, ${ }^{2}$ Luz Gonzalez-Hernandez, 'Moises Ramos Solano, ${ }^{2}$ Jaime AndradeVillanueva. 'Universidad de Guadalajara, Guadalajara, Mexico; ${ }^{2}$ Hospital Civil Fray Antonio Alcalde, Unidad De VIH, Guadalajara, Mexico

\subsection{6/sextrans-2019-sti.331}

Background Antiretroviral Therapy (ART) inhibits HIV replication, allowing immune reconstitution; however, some patients have an insufficient reconstitution, resulting in high levels of immune activation, microbial translocation, inflammation and intestinal dysbiosis. Since microbiota and its metabolites, such as Short-Chain Fatty Acids (SCFA) are linked to immune status, alteration of one or both could be related to poor $\mathrm{T}$ cells reconstitution.

Methods HIV+ patients with ART, grouped as immunologic responders, IRs: $>350$ cells $(n=18)$, immunologic non-responders, INRs: $<350$ cells $(n=17)$ and, healthy controls $(n=$ 14) were recruited. Absolute quantification of Firmicutes, Bacteroidetes, Actinobacteria, Proteobacteria, Bifidobacterium, Clostridium leptum, Clostridium coccoides, Lactobacillus and Faecalibacterium prausnitzii were measured in stool by qPCR. Levels of butyrate, propionate and acetate were quantified in stool by HPLC. Absolute CD4 nadir count, CD4/CD8 proportion and co-expression of HLA-DR/CD38 were determined by flow cytometry. Comparisons between groups were performed with Kruskal-Wallis test.

Results No differences were found in the four main phyla. Regarding probiotics, there were no differences in Lactobacillus and C. coccoides; however, IRs have less copies of C. leptum and $F$. prausnitzii in comparison to the other groups. In contrast, INRs presented similar amounts of probiotics as the healthy subjects. No differences were found in SCFA levels, except for acetate which was increased in IRs. Concerning the immune status, there was no difference in CD4 nadir; however, IRs had greater variation in this count and, significantly higher CD4/CD8 proportion. Whereas, co-expression of CD4 + HLA-DR+CD38+ was decreased in INRs in comparison with IRs.
Conclusion Immune status from HIV + subjects directly affects microbiota composition, systemic activation and inflammation. The enrichment of some probiotics, particularly SCFA-producing bacteria, is related to deficient immune reconstitution; however, other gut bacteria could be compensating this decrease. Further studies are necessary to understand how the microbiota and their metabolic products are related to CD4 T cells recovery.

Disclosure No significant relationships.

\section{P173 INVESTIGATING VARICELLA-ZOSTER VIRUS-SPECIFIC T CELLS THROUGH THE LENSES OF HIV}

${ }^{1}$ Carolina Moreira*, ${ }^{2}$ Catia Perciani, ${ }^{3}$ Thomas Murooka, ${ }^{4}$ Walter Jaoko, ${ }^{1}$ Kelly Macdonald, ${ }^{4}$ KAVI-ICR Team. ${ }^{1}$ University of Manitoba, Section of Infectious Diseases, Department of Internal Medicine, Winnipeg, Canada; ${ }^{2}$ University of Toronto, Department of Immunology, Toronto, Canada; ${ }^{3}$ University of Manitoba, Department of Immunology, Winnipeg, Canada; ${ }^{4}$ University of Nairobi, Kenya AIDS Vaccine Initiative (KAVI), Nairobi, Kenya

\subsection{6/sextrans-2019-sti.332}

Background Varicella-zoster virus (VZV), also known as chickenpox virus, constitutes a promising vector for a successful HIV vaccine. As an effort to scrutinize its potential, we are characterizing the susceptibility of VZV-specific CD4 T cells to HIV infection and the phenotypic profile of both CD4 and CD8 T cells.

Methods Blood T cells isolated from a cohort of healthy Kenyan women with pre-immunity to VZV (NCT02514018) were stimulated in vitro using 15 -mer peptides representing VZV glycoprotein E (gE) and VZV Open Reading Frame 4 (ORF4). CD4 and CD8 T cell memory phenotypes were characterized by flow cytometry based on the expression of CCR7/ CD45RA. The activation status of VZV-specific CD4 T cells was measured by the expression of HLA-DR, CD69, and CD25 after 6-day stimulation with gE and ORF4 peptides. Susceptibility to HIV infection was assessed using in vitro infection with a CCR5-tropic virus. DMSO and CMV peptides were used as negative and positive controls, respectively.

Results A similar frequency of central memory CD4 T cells $\left(\mathrm{T}_{\mathrm{CM}}\right)$ (median 24\%, IQR 18\%-32\%) and effector memory CD4 T cells ( $\left.\mathrm{T}_{\mathrm{EM}}\right)$ (median 27\%, IQR 20\%-32\%) was observed in our cohort. The predominant CD8 memory subtype was $\mathrm{T}_{\text {EMRA }}$ (median 28\%, IQR 21\%-40\%) followed by $\mathrm{T}_{\mathrm{EM}}$ cells (median 12\%, IQR 8\%-19\%) ( $\left.\mathrm{n}=45\right)$. Preliminary results show our ability to expand VZV-specific cells in culture using $\mathrm{gE}$ and ORF4 as stimuli and that these cells highly express the marker HLA-DR. Their susceptibility to in vitro $H I V$ infection is currently under investigation using CMV-specific cells as comparator.

Conclusion A viral vector able to sustain CD8 $\mathrm{T}_{\mathrm{EM}}$ responses without fueling the immune system with HIV target cells constitutes an ideal candidate for an HIV vaccine. Hence, our study sheds light on key aspects of VZV-specific immunity that will help determining its future as a vector in an HIV vaccine.

Disclosure No significant relationships. 